\title{
Do children with optic pathway tumors have an increased frequency of other central nervous system tumors?
}

\author{
David N. Korones, ${ }^{1}$ Jeannie Padowski, Brad A. Factor, and Louis S. Constine \\ University of Rochester School of Medicine and Dentistry, Rochester, NY 14642, USA
}

We questioned whether children with optic pathway tumors (OPTs) have an increased frequency of other CNS tumors on the basis of experience with a number of such children treated at our institution. The medical records of all patients with OPTs treated at Golisano Children's Hospital at Strong at the University of Rochester from 1957 to 2000 were reviewed to determine the incidence of additional CNS tumors in these children and whether the occurrence of these other CNS tumors is associated with any risk factors. Twenty-six children had OPTs. Twelve of the 26 children had biopsy-proved tumors; the remaining 14 were diagnosed on the basis of CT or MRI scans. Eight of the 26 patients $(31 \%)$ had a total of 11 additional CNS tumors. (One child had 2 additional CNS neoplasms, and a second child had 3.) Nine were biopsy proved ( 3 glioblastoma, 3 anaplastic astrocytoma, 3 low-grade astrocytoma), and 2 were diagnosed by imaging studies alone (acoustic neuromas). Eight of the 11 tumors occurred at a median of 5 years (0.8-25 years) subsequent to the diagnosis of the OPTs; 3 were diagnosed simultaneously with the OPT. Of the 17 children with neurofibromatosis (NF) and OPTs, $8(47 \%)$ had additional CNS tumors, while none of the 9 children $(0 \%)$ without NF had other CNS tumors $(P=0.023)$. There was no association between radiation treatment of the primary OPT and subsequent development of other CNS tumors in the group as a whole, or when the analysis was confined to children with NF.

Received July 22, 2002; accepted October 8, 2002.

${ }^{1}$ Address correspondence and reprint requests to David N. Korones, Golisano Children's Hospital at Strong, 601 Elmwood Avenue, Rochester, NY 14642 (david_korones@urmc.rochester.edu).

${ }^{2}$ Abbreviations used are as follows: $\mathrm{AA}$, anaplastic astrocytoma; $\mathrm{CP}$, cerebellopontine; GBM, glioblastoma multiforme; NF, neurofibromatosis; NF1, neurofibromatosis type 1; OPT, optic pathway tumor; $\mathrm{RR}$, relative risk; $\mathrm{RT}$, radiation therapy.
Despite this lack of statistical association, all 7 CNS tumors that occurred following radiation arose in irradiated areas. The risk of simultaneous or subsequent CNS tumors in children with NF and OPTs is high. These children should be closely monitored for the simultaneous or subsequent occurrence of other CNS tumors. Neuro-Oncology 5, 116-120, 2003 (Posted to NeuroOncology [serial online], Doc. 02-030, February 4, 2003. URL http://neuro-oncology.mc.duke.edu)

$\mathrm{T}$ he evaluation and management of children with optic pathway tumors (OPTs) ${ }^{2}$ has long been a vexing problem. These tumors are often difficult to biopsy without affecting vision, and they are capricious in their behavior, sometimes quite aggressive, sometimes quiescent for years. The tumors involve optic nerve(s), chiasm, and/or hypothalamus, and their behavior varies depending on location. Up to $50 \%$ of children with an OPT have neurofibromatosis (NF) (Cohen and Rothner, 1989; Oxenhandler and Sayers, 1978; Rush et al., 1982; Wong et al., 1987), and the behavior of this tumor and the risks of therapy may be different for children with NF than for children with OPT only.

Children with NF type1 (NF1) are at increased risk of developing malignancies, particularly central or peripheral nervous system tumors. The most common CNS tumor in patients with NF1 is OPT, occurring in approximately $5 \%$ to $15 \%$ of these children (Cohen and Rothner, 1989; Debella et al., 2000; Kuenzle et al., 1994; Listernick et al., 1989). The underlying cause of NF1, mutation of the putative tumor suppressor gene neurofibromin, may explain why children with NF1 are predisposed to developing malignancies (Reed and Gutmann, 2001). Children with OPTs and NF1 may be at risk of developing other tumors, and treatment of these children with radiation might heighten their risk of other CNS tumors in the field of radiation.

We reviewed the course of children who were treated 
for OPTs at Golisano Children's Hospital at Strong in Rochester, New York, from 1957 to 2000. The purpose of this review was (1) to determine the incidence of concomitant or subsequent CNS tumors in children with OPT, (2) to determine whether these additional tumors are more likely to occur in children with NF1, and (3) to determine whether treatment of these tumors with radiation heightens the risk of second CNS tumors.

\section{Methods}

Children followed for OPTs at Golisano Children's Hospital at Strong were identified through the databases of the Division of Pediatric Hematology/Oncology and Department of Radiation Oncology, the Strong Memorial Hospital Office of Clinical Practice Evaluation, and the Strong Memorial Hospital Tumor Registry. Children $\leq 18$ years of age diagnosed between 1957 and 2000 were included.

The medical records of children with OPTs were reviewed, and data on demographics, clinical presentation, means of diagnosis, treatment, and outcome were recorded. Children were considered to have OPTs if the tumors occurred in the optic nerve or chiasm, with or without extension into the hypothalamus, and if the tumors were either biopsy proved or documented by findings of thickened optic nerve(s) or chiasm or findings of a mass arising from the optic nerve(s) or chiasm on MRI or CT imaging. Additional CNS malignancies were defined as biopsy-proved brain tumors diagnosed either concurrently with the optic glioma or at any time subsequent to the diagnosis of the optic glioma. Two patients with presumed acoustic neuromas diagnosed by characteristic imaging studies alone were also included. In both instances the tumors were located at the cerebellopontine (CP) angle, and they were associated with hearing loss and increase in tumor size on serial CT scans. Additional CNS tumors diagnosed after the second tumors (i.e., third and fourth CNS tumors) were also included.

Proportions were compared by using the Fisher exact test. Overall survival and risk of developing subsequent CNS tumors were measured by the Kaplan-Meier method, and standard errors were calculated by the method of Greenwood. The log-rank test was used to determine any differences between groups in the risk of developing a subsequent CNS tumor (Altman, 1991). Statistical analyses were performed with the InStat program (GraphPad Software, San Diego, California).

\section{Results}

\section{Patients with OPTs}

Twenty-six children were diagnosed with OPTs between 1957 and 2000. Their median age at presentation was 5 years (range $0.5-12$ years). Eighteen $(69 \%)$ were female. Seventeen $(65 \%)$ had NF1. The OPT was biopsy proved in 12 children $(46 \%)$ and was diagnosed on the basis of imaging studies in 14 (54\%). Of the 12 biopsy-proved patients, 10 had a low-grade astrocytoma and 1 each had intermediate and high-grade astrocytomas. Two children had OPTs confined to the optic nerve, 12 that involved the optic nerve and chiasm, and 12 that involved the optic nerve, chiasm, and hypothalamus. Twenty-four children were initially evaluated because of symptoms referable to the tumor; 2 children with NF1 were diagnosed with an OPT on the basis of a surveillance MRI scan.

Treatment of the OPTs was variable. Eight children were initially observed, 9 were treated with radiation, 5 had initial surgery followed by radiation, and 1 each had radiation plus chemotherapy, resection plus chemotherapy, resection alone, and resection plus radiation plus chemotherapy. Nine of the 26 children $(35 \%)$ had recurrence or progression of their OPT. The 5 -year and 10 -year overall survival rates were $88 \% \pm 7 \%$ and $78 \% \pm 9 \%$, respectively (median follow-up 10.5 years; range $0.5-36$ years). Of the 6 children who died, 3 died of second CNS tumors, one died of toxicity from treatment of a second CNS tumor, 1 died because of progression of the OPT, and one died of unknown cause.

\section{Second CNS Tumors}

Eight of the 26 patients (31\%) had a total of 11 additional CNS tumors. Three patients had second CNS tumors at the time of presentation of the OPT. Second CNS tumors in the remaining 5 patients occurred at a median of 7 years. Median follow-up for the 8 children with additional CNS tumors was 6 years (range 1-25 years). Median follow-up for the other 18 children was 8 years (range $1-36$ years) $(P=0.8)$. Diagnoses of the additional CNS tumors included glioblastoma multiforme (GBM) (3), anaplastic astrocytoma (AA) (3), and low-grade astrocytoma (3). Two tumors were not biopsy proved. They were categorized as acoustic neuromas on the basis of location, documented growth, and associated hearing loss. Details on each of the 8 patients are listed in Table 1.

\section{Risk Factors for Second CNS Tumors}

Of the 17 children with NF1, $8(47 \%)$ had additional CNS tumors, while none of the 9 children $(0 \%)$ who did not have NF1 had other CNS tumors $(P=0.023)$. There was no difference between the length of follow-up from the time of diagnosis of the OPT for the children with NF1 (median follow-up 11 years, range 1-29 years) and the length of follow-up for the children who did not have NF1 (median follow-up 8 years, range $1-36$ years) $(P=$ $0.4)$.

There was no association of subsequent CNS tumors with prior treatment with radiation: Five of the 19 children $(26 \%)$ who were treated with radiation developed subsequent CNS tumors, while only one of the 7 children $(14 \%)$ who were not treated with radiation had a subsequent $\mathrm{CNS}$ tumor $(P=0.9)$. (Although 8 children with OPT had other CNS tumors, only 6 of these children had their other CNS tumors subsequent to the OPT. The CNS tumors in the remaining 2 children occurred simultaneously with their OPT; thus these 2 children were included 
Table 1. Patients with second CNS tumors: Timing of occurrence, diagnoses, history of NF, and any prior therapy for the original OPT

\begin{tabular}{|c|c|c|c|c|c|c|}
\hline Patient & $\begin{array}{l}\text { Years from } \\
\text { OPT to } \\
\text { additional } \\
\text { tumor }\end{array}$ & $\begin{array}{l}\text { Histology of additional } \\
\text { CNS tumor }\end{array}$ & $\begin{array}{l}\text { Site of } \\
\text { CNS tumor }\end{array}$ & NF & $\begin{array}{l}\text { Prior } \\
\text { RT }\end{array}$ & $\begin{array}{l}\text { Prior } \\
\mathrm{CT}\end{array}$ \\
\hline 1 & 0.8 & AA & Basal ganglia & Yes & Yes & No \\
\hline \multirow[t]{3}{*}{$2^{a}$} & 4 & Low-grade astrocytoma & Cerebellar vermis & Yes & Yes & No \\
\hline & 5 & Low-grade astrocytoma & Corpus callosum & & Yes & No \\
\hline & 7 & GBM & Temporal lobe & & Yes & No \\
\hline 3 & 25 & GBM & $\mathrm{CP}$ angle & Yes & Yes & No \\
\hline 4 & 0 & AA & $\begin{array}{l}\text { Basal ganglia, } \\
\text { Thalamus }\end{array}$ & Yes & $\mathrm{No}^{\mathrm{c}}$ & No \\
\hline 5 & 23 & Acoustic neuroma $^{\mathrm{b}}$ & $8^{\text {th }}$ nerve & Yes & Yes & No \\
\hline 6 & 7 & Acoustic neuroma $^{\mathrm{b}}$ & $8^{\text {th }}$ nerve & Yes & $\mathrm{No}^{\mathrm{d}}$ & No \\
\hline \multirow[t]{2}{*}{$7^{\mathrm{a}}$} & 0 & $\mathrm{AA}$ & $4^{\text {th }}$ ventricle & Yes & $\mathrm{No}^{\mathrm{c}}$ & No \\
\hline & 6 & GBM & Temporal lobe & & Yes & No \\
\hline 8 & 0 & Low-grade astrocytoma & Tectal plate & Yes & No & No \\
\hline
\end{tabular}

Abbreviations: AA, anaplastic astrocytoma; CP, cerebellopontine; CT, chemotherapy; GBM, glioblastoma multiforme; NF, neurofibromatosis; OPT, optic pathway tumor; RT, radiotherapy. apatient \#2 had third and fourth CNS tumor. Patient \#7 had third CNS tumor.

${ }^{\mathrm{b}}$ Tumors were not biopsy proved.

${ }^{c}$ Child had second CNS tumor simultaneously with OPT and prior to any radiation.

${ }^{\mathrm{d}}$ Child was not irradiated and had second CNS tumor subsequent to OPT.

in the group of children who did not have subsequent CNS tumors.) When the analysis was confined to children with NF1, 5 of 12 children $(42 \%)$ treated with radiation had subsequent CNS tumors, and 1 of the 5 children with NF1 $(20 \%)$ not treated with radiation had a subsequent $\mathrm{CNS}$ tumor $(P=0.6)$.

When the risk of subsequent CNS tumors over time was analyzed, there was still no significant difference between children who did or did not receive radiation therapy $(P=0.96$; log-rank test $)$ (Fig. 1). At 6-year follow-up, the risk of developing a subsequent CNS tumor was $25 \% \pm 21 \%$ in the radiated children versus $17 \% \pm 9 \%$ in the nonirradiated children $(P=0.96)$. When the analysis was confined to children with NF, there was still no difference between the irradiated and nonirradiated groups.

Although there was not a statistically significant association of second tumors with radiation of the primary OPTs, all 7 of the second tumors that occurred following radiation occurred in sites exposed to at least some radiation (Table 2). Three tumors occurred at sites receiving $\geq 40 \mathrm{~Gy}, 2$ tumors occurred at sites receiving 20-40 Gy, and 1 tumor occurred at a site receiving 10-30 Gy. The seventh tumor occurred in an irradiated volume, but the medical record is not available for review.

\section{Discussion}

In this series of 26 children with OPTs, a surprisingly high proportion of the children were found to have other CNS tumors, either simultaneously with or subsequent to presentation with the optic glioma. Furthermore, all 8 of the children with other CNS tumors had an underlying diagnosis of NF. The additional tumors in these children were often aggressive. Of the 6 children in this series who

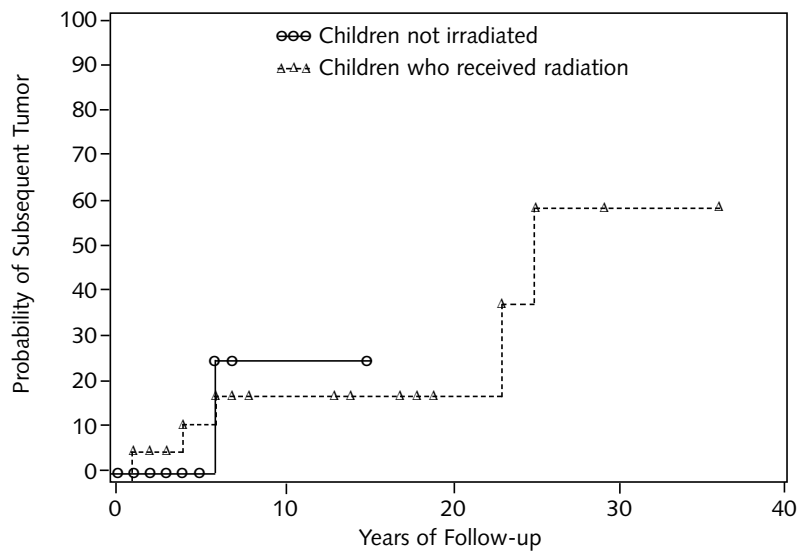

Fig. 1. The risk of developing a subsequent CNS tumor in irradiated versus nonirradiated children with optic pathway tumors.

died, 4 died not of the OPT, but of the other CNS tumor or of complications of treating that tumor.

The underlying defect in NF1 is mutation of the NF1 gene, a presumed tumor suppressor gene (Reed and Gutmann, 2001). This mutated tumor suppressor gene may explain the well-documented increased risk of malignancies in children with NF1. These children are at increased risk of acute myelogenous leukemia; solid tumors such as neurofibrosarcoma, rhabdomyosarcoma, and pheochromocytoma; and brain tumors, including OPTs, other astrocytomas, ependymoma, and meningioma (Cohen and Rothner, 1989). OPT is the most common tumor in these children, occurring in approximately $5 \%$ to $15 \%$ of children with NF1 (Cohen and Rothner, 1989; Debella et al., 2000; Kuenzle et al., 1994; Listernick et al., 1989). Treatment of this often indolent tumor 
Table 2. Second CNS tumors in children with NF1 treated with radiation: Age at diagnosis of second tumor, histology and location of second tumor, and dose of prior radiation at the site of the second tumor

\begin{tabular}{|c|c|c|c|c|c|c|c|c|}
\hline Patient & OPT location & $\begin{array}{l}\text { Radiation } \\
\text { dose (Gy) }\end{array}$ & $\begin{array}{l}\text { Age } \\
\text { (years) at } \\
\text { radiation } \\
\text { therapy }\end{array}$ & $\begin{array}{l}\text { Age } \\
\text { (years) at } \\
\text { second } \\
\text { CNS tumor }\end{array}$ & $\begin{array}{l}\text { Location of } \\
\text { second } \\
\text { CNS tumor }\end{array}$ & $\begin{array}{l}\text { Histology of } \\
\text { second } \\
\text { CNS tumor }\end{array}$ & $\begin{array}{l}\text { In } \\
\text { irradiated } \\
\text { volume? }\end{array}$ & $\begin{array}{l}\text { Estimated } \\
\text { dose at site } \\
\text { of second } \\
\text { tumor (Gy) }\end{array}$ \\
\hline 1 & Optic chiasm & 50.4 & 8.1 & 8.9 & Midbrain & AA & Yes & 50.4 \\
\hline \multirow[t]{3}{*}{2} & $\begin{array}{l}\text { Right optic nerve } \\
\text { and optic chiasm }\end{array}$ & 54 & 2 & 6 & Cerebellar vermis & $\begin{array}{l}\text { Low-grade } \\
\text { astrocytoma }\end{array}$ & Yes & $20-40$ \\
\hline & & & & 7 & $\begin{array}{l}\text { Splenium of corpus } \\
\text { callosum }\end{array}$ & $\begin{array}{l}\text { Low-grade } \\
\text { astrocytoma }\end{array}$ & Yes & $20-30$ \\
\hline & & & & 9 & Right temporal lobe & GBM & Yes & $40-53$ \\
\hline 3 & Left optic nerve & 45 & 3 & 28 & Left CP angle & GBM & Yes & $10-30$ \\
\hline 5 & $\begin{array}{l}\text { Optic nerves and } \\
\text { optic chiasm }\end{array}$ & 49 & 9 & 29 & Right $\mathrm{CP}$ angle & $\begin{array}{l}\text { Acoustic } \\
\text { neuroma }^{\mathrm{a}}\end{array}$ & Yes & 49 \\
\hline 7 & $\begin{array}{l}\text { Left optic nerve } \\
\text { and optic chiasm }\end{array}$ & 55 & 6 & 12 & Right temporal lobe & GBM & Yes & Unknown \\
\hline
\end{tabular}

Abbreviations: $\mathrm{AA}$, anaplastic astrocytoma; $\mathrm{CP}$, cerebellopontine; $\mathrm{GBM}$, glioblastoma multiforme, OPT, optic pathway tumor.

${ }^{a}$ Not biopsy proved.

has evolved over the past several decades from aggressive surgery and radiation, with or without chemotherapy, to more expectant treatment, reserving intervention for those children with documented growing tumors and/or visual loss.

As noted earlier, up to $50 \%$ of children with OPTs have NF (Cohen and Rothner, 1989; Oxenhandler and Sayers, 1978; Rush et al., 1982; Wong et al., 1987). In our series, $65 \%$ of the children had NF, a somewhat higher proportion than noted in other reports. We are not certain why so many of our patients with OPTs have NF. We are not a major referral center for this disease, and only 2 of the 17 children with NF1 in this study had the OPT detected by surveillance imaging. It is possible that this is chance variation due to small numbers of patients.

In most series of children with OPTs, there is little attention given to the simultaneous or subsequent occurrence of other CNS tumors, and when it is mentioned, the numbers reported are few (Danoff et al., 1980; Horwich et al., 1985; Oxenhandler and Sayers, 1978; Rush et al., 1982; Wong et al., 1987). However, in 2 recent reports, the incidence of other CNS tumors in this setting was surprisingly high. Kuenzle et al. (1994) reported on 21 children with NF1 and OPTs, noting that in 20-year follow-up, $11(52 \%)$ had second CNS tumors found outside the optic pathway. In contrast to our series, only 2 of the tumors were biopsy proved (WHO grade I astrocytoma), and only 1 was aggressive and lethal (a rapidly growing brainstem tumor). Friedman and Birch (1997) also noted an association between OPTs and other CNS tumors. Of 154 children with NF1 and OPTs, 17 (11\%) had second CNS tumors, whereas only $1.5 \%$ of NF1 patients without OPTs developed a CNS tumor. Their data suggest that patients with NF1 and OPTs are at higher risk of having other CNS tumors than unaffected NF1 patients.

Two of our patients had non-biopsy-proved acoustic neuroma, a tumor more typically associated with NF type 2. Despite this, both our patients had optic gliomas, and both had the clinical criteria for a diagnosis of NF1.
Although it is not common, acoustic neuroma has been reported in children with NF1 (Michels et al., 1989; White et al., 1983). We included these children in this series because both children had previously well-documented optic gliomas, their second tumors had progressed and were in the typical location for acoustic neuromas, and both patients had clinical presentations consistent with this tumor. Because these tumors are difficult to resect, they are often treated without obtaining a biopsy, as was the case with our patients. Should these two patients be removed from the analysis, the incidence of second tumors in patients with NF1 and OPTs is still high $(25 \%)$, and there is still a statistical association of second CNS tumors after OPT tumors with NF1.

A major concern in treating children with NF1 and OPTs is whether treatment with radiation might predispose these children to second malignancies in the irradiated volume: Because NF1 is caused by a mutation in a tumor suppressor gene, the concern is that the "second hit" caused by radiotherapy might heighten the risk of radiation-induced malignancies. This well-founded concern has been documented in other tumor suppressor syndromes, most notably the very high risk of radiationinduced second malignancies in children with familial retinoblastoma (Halperin et al., 1999).

We were unable to document an association between radiation and second CNS tumors in our series. The fact that 4 children had other CNS tumors prior to receiving radiation suggests that factors other than radiation are at play. Furthermore, Friedman and Birch (1997) point out that only 3 of their 17 patients with second CNS tumors received prior radiation, and that when these patients were removed from their analysis, they still found a significantly increased risk of other CNS tumors in patients with NF1 and OPTs compared with patients with NF1 alone. Kuenzle et al. (1994) do not report whether the NF1 patients with OPTs and second CNS tumors received radiation.

Although there was not a statistically significant association of radiation and additional CNS tumors in our series, all 7 of the CNS tumors occurring after radiation 
occurred in an irradiated volume. Thus it is quite possible that radiation heightens the risk of second CNS tumors in NF1 patients and that our small numbers account for the lack of a statistically significant association. A larger group of patients, which included patients with NF1 and OPTs who had undergone chemotherapy, radiotherapy, or observation, would be required to answer this question. Our small series precludes any firm conclusions regarding radiation as a risk factor for other
CNS tumors in children with NF1.

In sum, we report a high incidence of second CNS tumors in children with OPTs, all of them occurring in children with NF1. Although we could not document a statistically significant association of these tumors with prior radiation, radiation may be a contributing risk factor. Children with NF1 and OPTs should be followed closely not only for activity of their OPT, but also for the possibility of developing other CNS tumors.

\section{References}

Altman, D.G. (1991) Practical Statistics for Medical Research. London: Chapman and Hall.

Cohen, B.H., and Rothner, A.D. (1989) Incidence, types, and management of cancer in patients with neurofibromatosis. Oncology 3, 23-30.

Danoff, B.F., Kramer, S., and Thompson, N. (1980) The radiotherapeutic management of optic nerve gliomas in children. Int. J. Radiat. Oncol. Biol. Phys. 6, 45-50.

DeBella, K., Szudak, J., and Friedman, J.M. (2000) Use of the National Institutes of Health criteria for diagnosis of neurofibromatosis 1 in children. Pediatrics 105, 608-614.

Friedman, J.M., and Birch, P. (1997) An association between optic glioma and other tumours of the central nervous system in neurofibromatosis type 1. Neuropediatrics 28, 131-132.

Halperin, E.C., Constine, L.S., Tarbell, N.J., and Kun, L.E. (1999) Retinoblastoma. In: Pediatric Radiation Oncology, 3rd ed. Philadelphia: Lippincott Williams \& Wilkins, pp. 126-162.

Horwich, A., and Bloom, H.C.G. (1985) Optic gliomas: Radiation therapy and prognosis. Int. J. Radiat. Oncol. Biol. Phys. 11, 1067-1079.

Kuenzle, C., Weissert, M., Roulet, E., Bode, H., Schefer, S., Huisman, T., Landau, K., and Boltshauser, E. (1994) Follow-up of optic pathway glioma in children with neurofibromatosis type 1. Neuropediatrics 25, 295-300.
Listernick, R., Charrow, J., Greenwald, M.J., and Esterly, N.B. (1989) Optic gliomas in children with neurofibromatosis type 1. J. Pediatr. 114, 788-792.

Michels, V.V., Whisnant, J.P., Garrity, J.A., and Miller, G.M. (1989) Neurofibromatosis type 1 with bilateral acoustic neuromas. Neurofibromatosis $2,213-217$.

Oxenhandler, D.C., and Sayers, M.P. (1978) The dilemma of childhood optic gliomas. J. Neurosurg. 48, 34-41.

Reed, N., and Gutmann, D.H. (2001) Tumorigenesis in neurofibromatosis: New insights and potential therapies. Trends Mol. Med. 7, 157-162.

Rush, J.A., Younge, B.R., Campbell, R.J., and MacCarty, C.S. (1982) Optic glioma: Long-term follow-up of 85 histopathologically verified cases. Ophthalmology 89, 1213-1219.

White, G.E, and Toland, J. (1983) Neurofibromatosis and secondary acoustic neuroma. Am. J. Optom. Physiol. Opt. 60, 625-629.

Wong, J.Y., Uhl, V., Wara, W.M., and Sheline, G.E. (1987) Optic gliomas. A reanalysis of the University of California, San Francisco experience. Cancer 60, 1847-1855. 